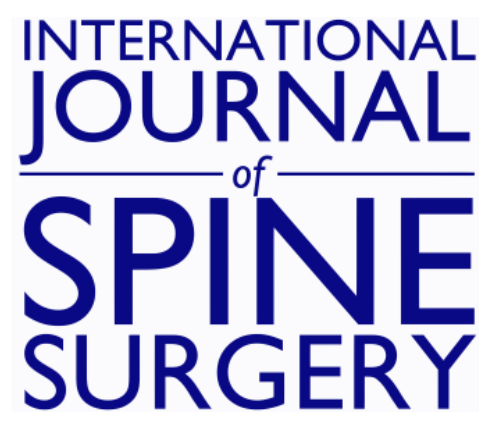

\title{
Management of Pyogenic Spondylodiscitis Following Nonspinal Surgeries: A Tertiary Care Center Experience
}

Ghazwan A. Hasan, Hayder Q. Raheem, Ahmed Qutub, Yasameen Bani Wais, Mustafa Hayder Katran and Gautam M. Shetty

Int J Spine Surg 2021, 15 (3) 591-599

doi: https://doi.org/10.14444/8080

http://ijssurgery.com/content/15/3/591

This information is current as of April 25, 2023.

Email Alerts Receive free email-alerts when new articles cite this article. Sign up at: http://ijssurgery.com/alerts 


\title{
Management of Pyogenic Spondylodiscitis Following Nonspinal Surgeries: A Tertiary Care Center Experience
}

\author{
GHAZWAN A. HASAN, MD, ${ }^{1}$ HAYDER Q. RAHEEM, MD,${ }^{2}$ AHMED QUTUB, $M D,{ }^{3}$ \\ YASAMEEN BANI WAIS, MD ${ }^{4}$ MUSTAFA HAYDER KATRAN, MD, ${ }^{2}$ GAUTAM M. SHETTY, MSOrth ${ }^{5,6}$ \\ ${ }^{I}$ Department of Orthopaedic Surgery, Al-Kindi Teaching Hospital, Baghdad, Iraq, ${ }^{2}$ Al-Mustansiria Medical College, Baghdad, Iraq, ${ }^{3}$ Imam Zain Alabdine \\ Hospital, Karbala, Iraq, ${ }^{4}$ Private Clinic, Baghdad, Iraq, ${ }^{5}$ Orthopaedic \& Knee Clinic, Mumbai, India, ${ }^{6}$ AIMD Research, Mumbai, India
}

\begin{abstract}
Background: The literature is lacking on the incidence and management of pyogenic spondylodiscitis (PS) following routine elective surgical procedures. This study aimed to analyze the presentation and treatment outcome in patients with PS following nonspinal surgeries at a tertiary care center with a minimum follow-up of 12 months.

Methods: The demographic, clinical-radiologic features, and treatment outcomes in 40 patients with a diagnosis of PS following nonspinal surgical procedures were retrospectively reviewed and analyzed.

Results: The mean age at presentation was $36.4 \pm 11.8$ years, with $80 \%$ of patients being female. The common surgical procedures associated with PS were cesarean delivery $(30 \%)$, gastric sleeve surgery $(12.5 \%)$, and dilatation and curettage $(12.5 \%)$. The tissue biopsy culture was positive in $82.5 \%$ of patients. A total of 26 patients $(65 \%)$ were treated with conservative management and 14 patients $(35 \%)$ were treated surgically. The mean pretreatment Core Outcome Measure Index score significantly decreased at 12 months $(P<.0001)$ after treatment. The mean pretreatment erythrocyte sedimentation rate $(P<.0001)$ and $C$-reactive protein $(P<.0001)$ levels significantly decreased at 12 months after treatment.

Conclusions: With most patients with PS following nonspinal surgeries treated with conservative management, excellent clinical outcomes were achieved in all patients at 12 months after treatment. The diagnosis of PS should be considered in patients presenting with low back pain (LBP) with a recent history of undergoing a nonspinal surgical procedure. Patients who undergo surgical procedures are an important "at-risk" patient population, and early diagnosis and treatment can help achieve excellent clinical outcomes. Further studies are required to determine risk factors and possible perioperative precautions that can be taken to prevent PS in patients who undergo nonspine surgeries.

Level of Evidence: 3.

Clinical Relevance: Pyogenic spondylodiscitis should be suspected in patients presenting with LBP after a recent non-spinal surgical procedure. Early diagnosis and treatment can help achieve excellent clinical outcomes in these patients.
\end{abstract}

Other \& Special Categories

Keywords: pyogenic spondylodiscitis, spinal infections, outcome, infection, surgery

\section{INTRODUCTION}

The incidence of pyogenic (nontubercular) spondylodiscitis (PS) has been reported to be up to 5.8 cases per 100000 person-years, with an average adjusted annual increase of $7.0 \% .{ }^{1}$ With the increase in the number of the elderly in the general population and the increased prevalence of comorbidities like diabetes, malignancies, and end-stage renal disease, the incidence of PS has risen over the years. ${ }^{1,2}$ Although PS following spinal procedures such as surgery or injections, secondary to immunecompromise, or due to hematogenous spread from an infective focus elsewhere in the body is well reported, ${ }^{3-5}$ PS secondary to elective surgical procedures is rare.
Few case reports of PS secondary to genitourinary surgical procedures, such as sacrohysteropexy, ${ }^{6}$ rectopexy, ${ }^{7}$ and transrectal prostate biopsy, ${ }^{8}$ or following major surgeries, such as abdominal aortic aneurysm repair ${ }^{9}$ and lung transplantation, ${ }^{10}$ have been reported previously. Surgical procedures are known to cause dysregulation of the immuneinflammatory response and reduce resistance to infection postoperatively. ${ }^{11}$ Furthermore, gynecologic and genitourinary surgical procedures are known to cause postoperative bacteremia despite prophylactic antibiotics, increasing the risk of bone and joint infection following these procedures. ${ }^{12,13}$ However, the literature is lacking on PS following routine elective surgical procedures, such as caesar- 
ean delivery, ligament reconstruction, and bariatric surgery, and the treatment outcome of such patients. Hence, this study aimed to analyze the presentation and treatment outcome in patients with PS following nonspinal surgical procedures with a minimum posttreatment follow-up of 12 months.

\section{METHODS}

\section{Study Population}

We retrospectively reviewed the records of patients who were treated for PS at the orthopaedic spine surgery department of a tertiary care center between September 2016 and April 2020. The inclusion criterion was all patients with a diagnosis of PS after evaluation (clinical, radiologic, and blood investigations) for low back pain (LBP). The exclusion criteria were PS following spine surgery or patients with a history of spine surgery, history of receiving epidural injection or block for LBP, patients with tubercular spondylodiscitis or spontaneous PS, and patients who did not have a minimum follow-up of 12 months. This study was approved by an institutional ethics committee and has been performed in accordance with the ethical standards as laid down in the 1964 Declaration of Helsinki and its later amendments or comparable ethical standards.

\section{Patient Evaluation}

All patients were evaluated with a thorough history and clinical examination at presentation. Demographic data, including sex, age, body mass index, comorbidities, details of past surgeries, or procedures, were collected from all patients. The algorithm proposed by Duarte and Vaccaro ${ }^{14}$ was followed in the current study to diagnose and treat PS, which was based on clinical, radiographic, and culture findings. The clinical diagnosis of PS was suspected when patients had symptoms such as nonspecific spinal pain not relieved with rest or pain medication, painful spinal range of motion, neurologic symptoms, such as sensory or motor deficits, associated fever (when present), and presence of associated comorbidities, such as diabetes and prolonged corticosteroid intake. ${ }^{2,15}$ Patients with suspected PS underwent blood investigation to include erythrocyte sedimentation rate (ESR) and $\mathrm{C}$-reactive protein (CRP) and magnetic resonance imaging (MRI) with contrast. In all patients with MRI evidence of vertebral infection (T1-weighted images showing decreased signal intensity in the vertebral body and disk and loss of end plates, and T2-weighted images showing increased signal intensity of the disk and vertebral body), ${ }^{16,17}$ subsequent blood culture and biopsy and culture of intradiscal tissue sample were performed. All patients also underwent a polymerase chain reaction test of the tissue sample to rule out the diagnosis of tuberculosis. The severity of PS was classified into 3 grades (grade I, II, or III) based on the clinical-radiologic scoring system proposed by Homagk et al. ${ }^{18}$ For the lumbar spine fluoroscopy-guided biopsies, and for cervical spine computed tomography (CT)-guided biopsies, were performed to collect tissue samples for culture.

\section{Treatment}

Conservative treatment of patients included bed rest for an initial 1 week, rigid bracing for 12 weeks, and antibiotic therapy for 12 weeks. For patients with positive biopsy cultures, intravenous (IV) antibiotic treatment was administered for the first 6 weeks followed by oral antibiotics for the next 6 weeks with the 2 most sensitive antibiotics according to culture sensitivity results. Intravenous and oral vancomycin at 15 to $20 \mathrm{mg} / \mathrm{kg}$ BID and IV ceftriaxone $1 \mathrm{~g}$ BID was the combination commonly used in these patients. For patients with negative biopsy culture, IV and oral antibiotics were administered for 12 weeks based on the blood culture (if positive) or empirically based on broad Gram-positive, Gram-negative, and anaerobic antibacterial coverage. Intravenous ciprofloxacin 500 $\mathrm{mg}$ BID, IV ceftriaxone $1 \mathrm{~g}$ BID, and oral levofloxacin $500 \mathrm{mg}$ OD was the combination commonly used in these patients. Surgical management was indicated in patients with neurologic deficit or patients with grade II or III PS with significant end plate destruction and features of instability on MRI during presentation, and nonresponse or failure of conservative treatment at the end of 6 weeks, especially in the presence of a negative biopsy culture. Surgical procedures performed as treatment included debridement with posterior pedicle screws fixation, or $360^{\circ}$ fusion in 2 stages (anterior followed by posterior), anterior cervical discectomy and fusion, or anterior cervical corpectomy and fusion (ACCF). All patients who underwent surgical management received 6 weeks of IV antibiotics followed by 6 weeks of oral antibiotics based on intraoperative tissue biopsy culture 
or empirical broad-spectrum antibiotics. Intravenous and oral vancomycin at 15 to $20 \mathrm{mg} / \mathrm{kg}$ BID and IV ceftriaxone $1 \mathrm{~g}$ BID was the combination commonly used in these patients. Response to treatment was monitored based on improving clinical symptoms and decreasing ESR and CRP levels during the follow-up period. Additional plain spine $\mathrm{x}$-rays were done in all surgical patients to evaluate improvement during the follow-up period.

\section{Outcome Measures}

All patients were followed up at 6 weeks, 3 months, 6 months, and 12 months after treatment. Clinical improvement was measured using the Core Outcome Measure Index (COMI) Score, which evaluated pain, function, generic health status or well-being, disability, and satisfaction, ${ }^{19}$ and ESR and CRP levels at each follow-up visit.

\section{Statistical Analysis}

Pretreatment and at 6 weeks, 3 months, 6 months, and 12 months posttreatment ESR and CRP levels and COMI scores were compared to determine the effectiveness of treatment. Subgroup analysis was performed to compare pretreatment and posttreatment (at 12 months) COMI, ESR, and CRP values in the subgroup treated conservatively versus the subgroup treated surgically. All categoric variables were compared using Fisher test and continuous variables were compared using $t$ test. A Pearson correlation (between 2 continuous variables) and a Spearman $\rho$ correlation (between 1 continuous and 1 categoric variable) analyses were performed to determine correlation between posttreatment COMI score (at 12 months) and pretreatment factors, such as age, sex, site of lesion (lumbar and nonlumbar), duration of symptoms, MRI grade, COMI, ESR, and CRP values. A $P$ value $<.05$ was considered statistically significant. Statistical analysis was performed using the GraphPad QuickCalcs online statistical analysis tool (GraphPad Software, San Diego, California).

\section{RESULTS}

\section{Patient Characteristics}

During the study period, a total of 70 patients received a diagnosis of PS. Of these 70 patients, 19 patients were excluded because of postspinal surgery PS, and 11 patients were excluded because of spontaneous (nonsurgical, spinal or nonspinal) PS.
Table 1. Demographic data of the study population.

\begin{tabular}{lc}
\hline Parameters & Value \\
\hline No. of patients & 40 \\
Age, mean \pm SD $(95 \%$ CI), y & $11.8(32.6-40.1)$ \\
Sex, n (\%) & \\
Male & $8(20)$ \\
Female & $32(80)$ \\
Clinical presentation, No. (\%) & $23(57.5)$ \\
Back pain & $5(12.5)$ \\
Back pain with radiculopathy & $10(25)$ \\
Neck pain & $2(5)$ \\
Neck pain with radiculopathy & \\
Comorbidities, n (\%) & $4(10)$ \\
Diabetes & $5(12.5)$ \\
Hypertension & $1(2.5)$ \\
Urinary tract infection & $2(5)$ \\
Steroid intake & $3(7.5)$ \\
Smoking & \\
Clinicoradiologic severity of PS, n (\%) & $28(70)$ \\
Grade I & $5(12.5)$ \\
Grade II & $7(17.5)$ \\
Grade III & $10.4(11.8-18.5)$ \\
Duration of presenting symptoms, & \\
mean \pm SD (95\% confidence & \\
interval), days & \\
\hline Abbreviation: PS, pyogenic spondylodiscitis. &
\end{tabular}

Hence, 40 patients of PS that occurred in patients who underwent nonspinal surgery were included and analyzed for this study. The demographic details of the study population are summarized in Table. 1. The mean age at presentation was $36.4 \pm$ 11.8 years, with $80 \%$ of patients being female. The mean onset of spine pain after nonspinal surgery was $15.2 \pm 10.4$ days (range, 5-60 days), with $57.5 \%$ of patients presenting with low back pain and $17.5 \%$ presenting with sensory and/or motor deficit in the upper or lower limbs (Table 1). Comorbidities were present in 37.5\% (15 of 40) of the patients (Table 1).

All patients received general anesthesia, and none of the patients reported any postoperative surgical site infection following their surgical procedure. The common surgical procedures associated with spondylodiscitis were cesarean delivery $(30 \%)$, gastric sleeve surgery $(12.5 \%)$, and dilatation and curettage (12.5\%; Table 2). The lumbar spine was the most commonly affected region $(77.5 \%)$, with a single level affected in $95 \%$ of patients and the L3 to L4 $(23 \%)$ and L4 to L5 (23\%) levels most commonly affected (Table 3). Based on the scoring system proposed by Homagk et al, ${ }^{18}$ there were 28 patients $(70 \%)$ with grade I PS, 5 patients $(12.5 \%)$ with grade II PS, and 7 patients $(17.5 \%)$ with grade III PS (Table 1 and Figure 1). The blood culture was positive in $42.5 \%$ of patients, and biopsy culture was positive in $82.5 \%$ of patients (Table 4 ). The common microorganism isolated was Staphylococ- 
Table 2. Details of previous surgery done in the study population.

\begin{tabular}{llc}
\hline Type of Surgery & \multicolumn{1}{c}{$\begin{array}{c}\text { Surgical } \\
\text { Wound Type }\end{array}$} & n (\%) \\
\hline Rhinoplasty & Clean contaminated & $2(5)$ \\
Laparoscopic cholecystectomy & Clean contaminated & $4(10)$ \\
Cesarean section & Clean & $12(30)$ \\
Abdominal hernia repair & Clean & $1(2.5)$ \\
Syme amputation of the & Contaminated & $1(2.5)$ \\
$\quad$ left foot & Clean & $1(2.5)$ \\
Decompressive drilling of the & & \\
$\quad$ hip joint & Clean & $1(2.5)$ \\
Knee ACL reconstruction & Clean contaminated & $5(12.5)$ \\
Gastric sleeve surgery & Clean & $2(5)$ \\
Diagnostic laparoscopy & Clean contaminated & $2(5)$ \\
Transvaginal biopsy & Clean & $1(2.5)$ \\
Breast lump excision & Contaminated & $5(12.5)$ \\
Dilatation and curettage & Clean contaminated & $2(5)$ \\
Tonsillectomy & Contaminated & $1(2.5)$ \\
Transurethral endoscopic & & \\
$\quad$ surgery for BPH & & \\
\hline
\end{tabular}

Abbreviations: ACL, anterior cruciate ligament; $\mathrm{BPH}$, benign prostate hyperplasia.

cus aureus in $20 \%$ of patients with positive blood culture, and $S$ aureus in $30 \%$ of patients and Escherichia coli in $20 \%$ of patients with positive tissue biopsy culture (Table 4).

A total of 26 patients $(65 \%)$ were treated with conservative management, and 14 patients $(35 \%)$ were treated surgically. Three patients were initially treated conservatively and were subsequently treated with surgery (debridement and posterior instrumentation). A total of 10 patients underwent debridement and posterior instrumentation, 2 pa-
Table 3. Magnetic resonance imaging findings in the study population.

\begin{tabular}{lc}
\hline Parameter & $\mathbf{n}(\mathbf{\%})$ \\
\hline Spinal regions involved & \\
Lumbar & $31(77.5)$ \\
Cervical & $8(20)$ \\
Thoracic & $1(2.5)$ \\
Patients with the number of spinal levels involved & \\
1 level & $38(95)$ \\
2 levels & $1(2.5)$ \\
3 levels & $1(2.5)$ \\
Specific spinal levels involved & \\
C4-C5 & $4(9)$ \\
C5-C6 & $5(11.5)$ \\
C6-C7 & $1(2.5)$ \\
D7-D8 & $2(5)$ \\
D12-L1 & $2(5)$ \\
L1-L2 & $1(2.5)$ \\
L2-L3 & $3(7)$ \\
L3-L4 & $10(23)$ \\
L4-L5 & $10(23)$ \\
L5-S1 & $5(11.5)$ \\
\hline
\end{tabular}

tients underwent anterior cervical discectomy and fusion, and 1 patient each underwent ACCF (Figure 2) and $360^{\circ}$ fusion.

\section{Treatment Outcomes}

The mean pretreatment COMI score of $7.3 \pm 0.7$ significantly decreased to $6.1 \pm 0.9(P<.0001)$ at 6 weeks, to $2.5 \pm 1.5(P<.0001)$ at 3 months, to 0.5 $\pm 0.6(P<.0001)$ at 6 months, and to $0.2 \pm 0.2(P$ $<.0001)$ at 12 months after treatment (Figure 3). The mean pretreatment ESR level significantly
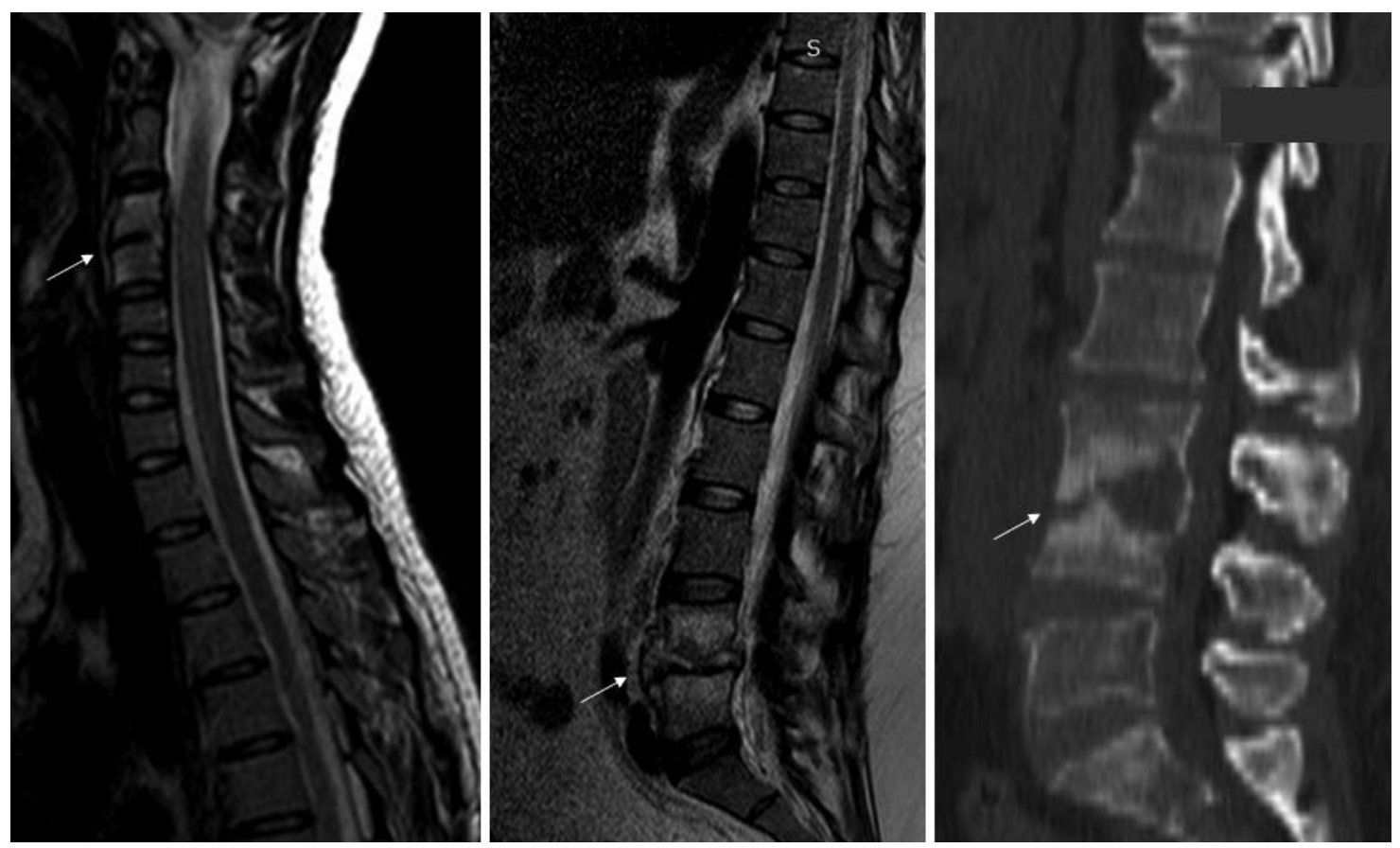

Figure 1. Example of grade I pyogenic spondylodiscitis (PS) at C3 to C4 level (arrow) on magnetic resonance imaging (MRI; left), grade II PS at L4 to L5 level (arrow) on MRI (center), and grade III PS at L3 to L4 level (arrow) on computed tomography scan (right). 

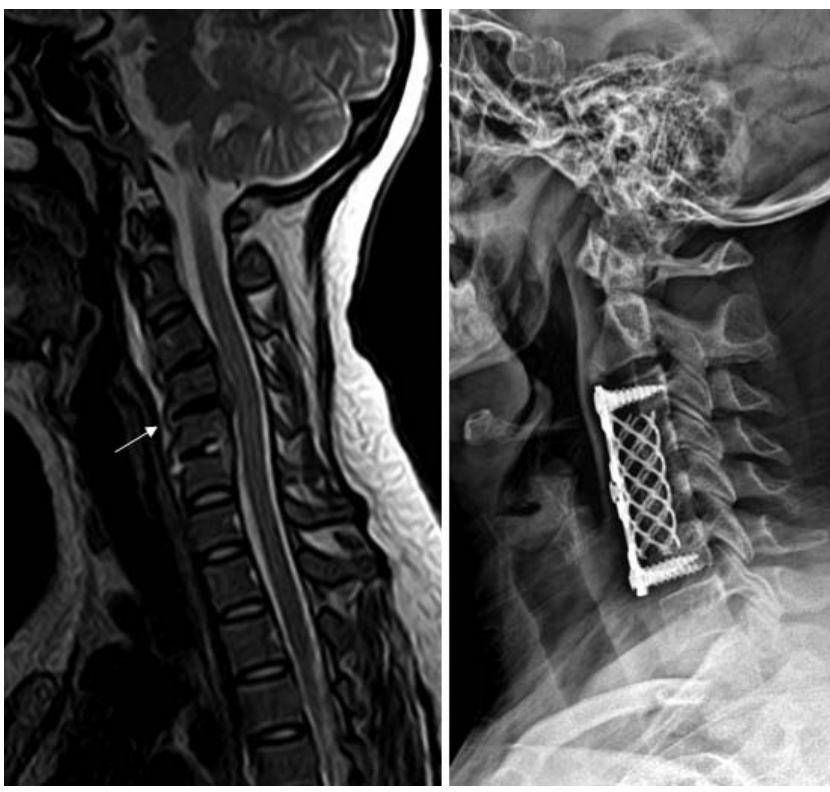

Figure 2. Preoperative cervical spine magnetic resonance image of a patient with grade III pyogenic spondylodiscitis at the C4 to C6 levels (left) and cervical spine lateral radiograph of the same patient at 12 months after anterior cervical corpectomy and fusion surgery (right).

decreased at 6 weeks $(P=.02), 3$ months $(P<$ $.0001), 6$ months $(P<.0001)$, and 12 months $(P<$ $.0001)$ after treatment (Figure 4). Similarly, the mean CRP value significantly decreased at 6 weeks $(P<.0001), 3$ months $(P<.0001), 6$ months $(P<$ $.0001)$, and 1 year $(P<.0001)$ after treatment when
Table 4. Details of culture findings in the study population.

\begin{tabular}{lc}
\hline Culture Findings & $\mathbf{n}(\mathbf{\%})$ \\
\hline Blood culture & \\
No growth & $23(57.5)$ \\
S aureus & $8(20)$ \\
MRSA & $2(5)$ \\
E coli & $4(10)$ \\
Burkholderia cepacia & $1(2.5)$ \\
Proteus sp & $1(2.5)$ \\
Klebsiella pneumoniae & $1(2.5)$ \\
Tissue biopsy culture & \\
S aureus & $12(30)$ \\
E coli & $8(20)$ \\
No growth & $7(17.5)$ \\
MRSA & $5(12.5)$ \\
B cepacia & $3(7.5)$ \\
Proteus sp & $3(7.5)$ \\
K pneumoniae & $1(2.5)$ \\
Brucella sp & $1(2.5)$ \\
\hline
\end{tabular}

Abbreviation: MRSA, methicillin-resistant $S$ aureus.

compared to the pretreatment value (Figure 4). There was no significant difference between posttreatment COMI, ESR, and CRP values at 12 months when the subgroup treated conservatively was compared to the subgroup treated surgically (Table 5).

\section{Correlation Analysis}

There was a weak but significant correlation between the site of lesion $(r=-0.31, P=.04)$ and posttreatment COMI value at 12 months. However,

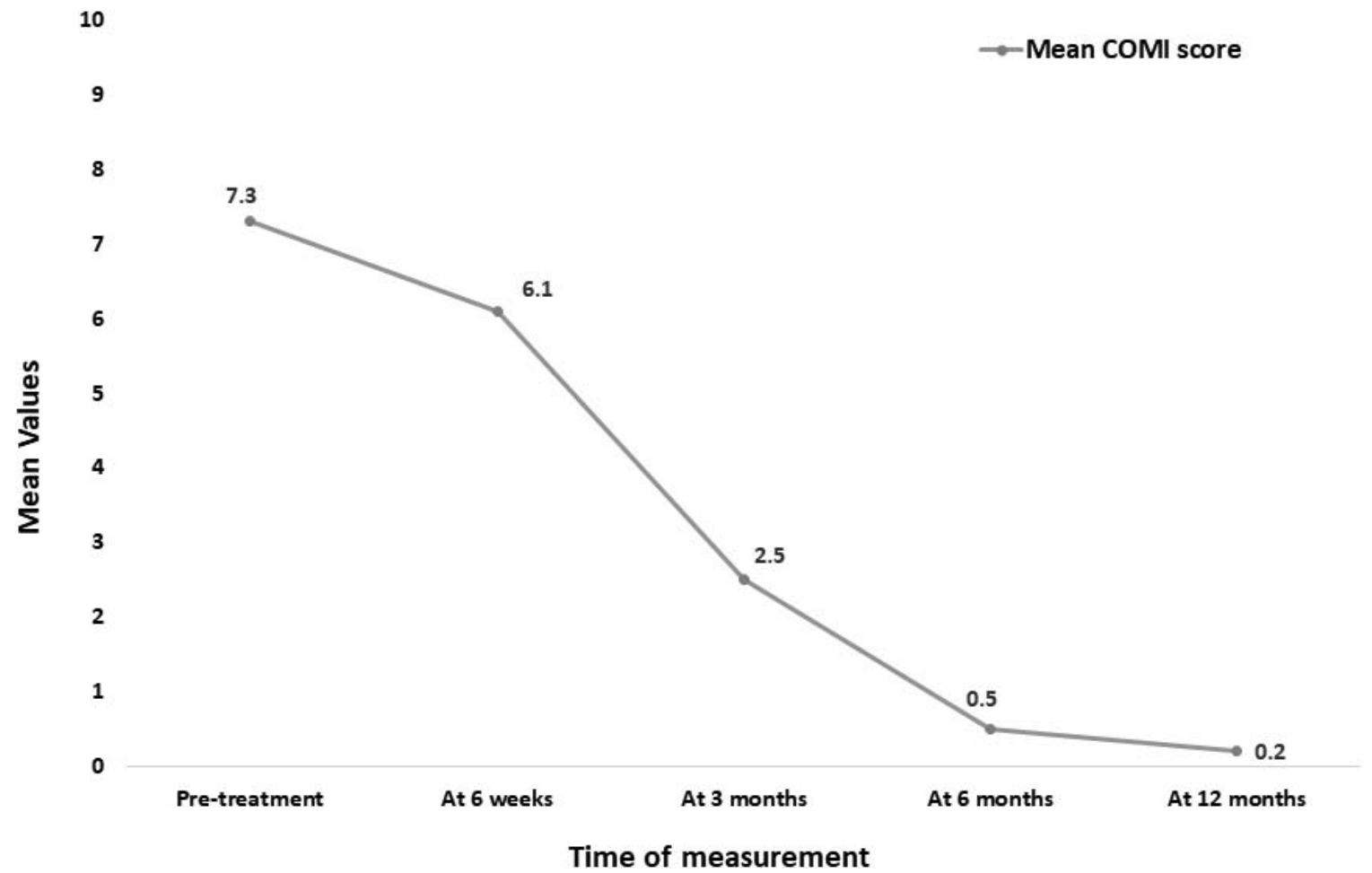

Figure 3. Mean Core Outcome Measure Index (COMI) scores at 6 weeks, 3 months, 6 months, and 12 months after treatment. 


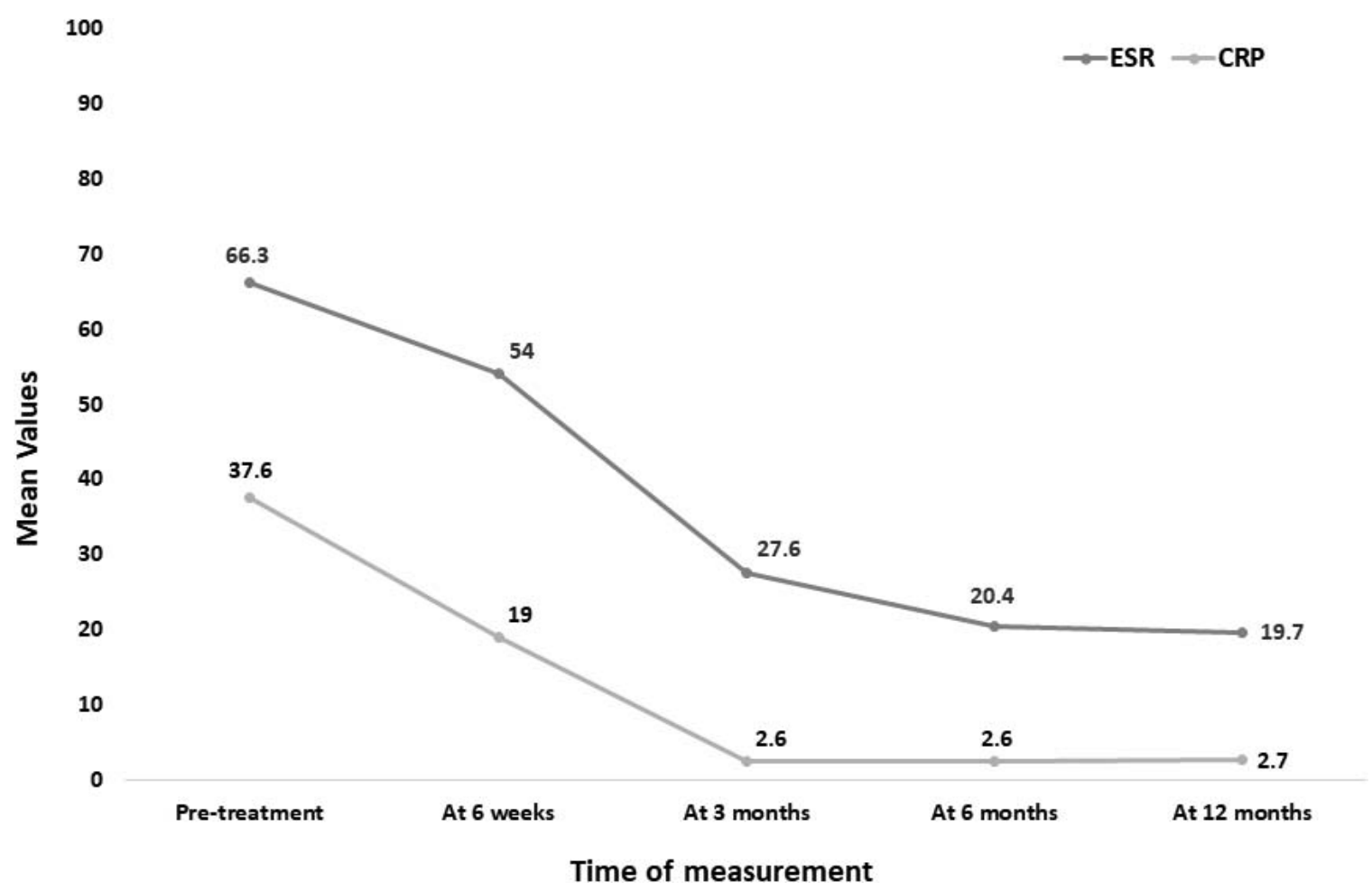

Figure 4. Mean erythrocyte sedimentation rate (ESR) and C-reactive protein (CRP) levels at 6 weeks, 3 months, 6 months, and 12 months after treatment.

no correlation was found between age $(r=-0.11, P$ $=.49)$, sex $(r=0.17, P=.27)$, duration of symptoms $(r=-0.00, P=1.00)$, MRI grade $(r=0.00, P=.97)$, pretreatment COMI $(r=-0.10, P=.53), \operatorname{ESR}(r=$ $-0.11, P=.49)$, CRP $(r=0.06, P=.71)$ values and posttreatment COMI value at 12 months.

\section{DISCUSSION}

The current case series aimed to analyze the presentation and treatment outcome in patients with PS following nonspinal surgical procedures with a minimum posttreatment follow-up of 12 months. The most common surgical procedure associated with PS was a cesarean delivery, the most common pathogen responsible was $S$ aureus, and the lumbar region was the most commonly affected area in this case series. Furthermore, with most patients treated with conservative management $(65 \%)$, excellent clinical outcomes were achieved in all patients, as indicated by sequential improvement in COMI scores and ESR and CRP levels at 6 weeks, 3 months, 6 months, and 12 months after treatment.

Pyogenic spondylodiscitis following nonspinal surgery is a rare condition with few cases published in the literature. ${ }^{6-10}$ Although PS following nonspinal surgery is typically associated with procedures that involve entry into the gastrointestinal or genitourinary tract or in immunocompromised patients or patients with serious comorbidities, ${ }^{6-10}$ the current case series included patients who had undergone a wide variety of surgical procedures, including procedures that are classified as class I or "clean" surgical wound (Table 2) based on the US Centers for Disease Control and Prevention classi-

Table 5. Comparison of Core Outcome Measure Index (COMI), erythrocyte sedimentation rate (ESR), and C-reactive protein (CRP) values between conservative and surgical treatment subgroups. ${ }^{a}$

\begin{tabular}{|c|c|c|c|}
\hline Parameters & Conservative Treatment & Surgical Treatment & $P$ Value \\
\hline No. of patients & 26 & 14 & - \\
\hline Pretreatment COMI score & $7.4 \pm 0.6(7.1-7.6)$ & $6.9 \pm 0.8(6.4-7.3)$ & .03 \\
\hline Pretreatment ESR level & $64.3 \pm 27.7(53.1-75.4)$ & $69.9 \pm 33(50.8-88.9)$ & .57 \\
\hline Pretreatment CRP level & $35.5 \pm 21(27.0-43.9)$ & $41.6 \pm 17.8(31.3-51.8)$ & .36 \\
\hline Posttreatment COMI score at 12 mo & $0.3 \pm 0.2(0.2-0.3)$ & $0.2 \pm 0.2(0.0-0.3)$ & .13 \\
\hline Posttreatment ESR level at 12 mo & $19.4 \pm 7.5(16.3-22.4)$ & $20.4 \pm 5.2(17.3-23.4)$ & .65 \\
\hline Posttreatment CRP level at $12 \mathrm{mo}$ & $2.8 \pm 1.5(2.1-3.4)$ & $2.5 \pm 0.5(2.2-2.7)$ & .47 \\
\hline
\end{tabular}

${ }^{\mathrm{a}}$ All data presented as mean \pm standard deviation $(95 \%$ confidence interval); $P<.05$ is considered statistically significant (bold). 
fication of surgical wounds. ${ }^{20}$ Most patients in the current series were female (male to female sex ratio of 1:4), with most of them undergoing gynecologic, obstetric, and gastrointestinal procedures. Furthermore, the median age of patients in the current series was 34 years (range, 18-65 years), with most $(82.5 \%)$ of the patients between ages 20 and 50 years. This is contrary to the findings of a previous report on PS by Pola et al, ${ }^{21}$ where the median age was 67 years and most of the patients were male (male to female sex ratio of 1.8:1). This could be explained by the fact that the underlying cause of PS in the study by Pola et $\mathrm{al}^{21}$ was secondary to hematogenous spread in patients with comorbidities or patients who were immunocompromised, or following spinal procedures.

The median duration for presentation after the onset of symptoms was 13 days (range, 5-60 days), and most of the patients in the current series presented with LBP $(57.5 \%), 25 \%$ of patients presented with neck pain with cervical spine involvement, and $17.5 \%$ of patients had a neurologic impairment. Pola et $\mathrm{al}^{21}$ in an analysis of 250 patients with PS reported a median duration for presentation after the onset of symptoms of 30 days (range, 15-63 days), with most of the patients presenting with LBP $(93.2 \%)$ and $23.5 \%$ of patients having a neurologic impairment. The greater duration of presentation, predominant lumbar spine affection, and a greater percentage of patients with neurologic impairment could be due to $23.4 \%$ of their cases occurring secondary to spine procedures. ${ }^{21}$ In the current series, although blood culture was positive in $42.5 \%$ of patients, tissue biopsy culture isolated the causative organism of PS in $82.5 \%$ of patients, with $S$ aureus the most common pathogen isolated in $30 \%$ of tissue biopsy culture (Table 4). These findings were similar to results published by Sur et al, ${ }^{22}$ where tissue culture biopsy was negative in $16.5 \%$ of patients and the most common isolated organism was $S$ aureus in patients with spontaneous PS.

Most patients $(65 \%)$ in the current series were treated conservatively, whereas $35 \%$ were treated with surgery. The clinical outcome measured by mean COMI score showed significant improvement during 3, 6, and 12 months after treatment. These results are similar to the results reported by Pandita et $a 1,{ }^{5}$ who reported significant improvement in the Oswestry Disability Index score in 11 patients with subacute, spontaneous PS at the end of 1 year where
$54 \%$ of patients underwent surgical treatment. There was a significant improvement in blood indicators of infection (ESR and CRP) at 3, 6, and 12 months of treatment in the current series (Figure 2). This was similar to improvements in ESR and CRP levels reported in previous studies following the treatment of PS. ${ }^{4,5,21}$ Furthermore, there was no significant difference in treatment outcomes in terms of the COMI, ESR, and CRP values at 12 months between the conservatively treated and surgically treated subgroups, indicating that conservative treatment had success rates similar to those of surgical treatment in patients with PS following nonspinal surgeries. These findings were similar to those in the study by Tschugg et $\mathrm{al}^{4}{ }^{4}$ who reported no significant difference in terms of numeric rating scale for pain, ESR, and CRP values at 12 months between subroups treated conservatively or surgically in patients with both primary acquired and postspinal surgery PS. Among pretreatment factors, such as age, sex, site of lesion (lumbar and nonlumbar), duration of symptoms, MRI grade, COMI, ESR, and CRP values, our study demonstrated a significant weak correlation between mean COMI score at 12 months and the site of lesion. Hence, patients with lumbar spine lesion were associated with lower COMI scores and better clinical outcome at 12 months. This could be because cervical spondylodiscitic lesions have been reported to be associated with more destructive disease and a higher risk of undergoing surgical treatment. $^{23}$

In the current series, most patients underwent abdominal, gynecologic, or obstetric surgeries (77.5\%; Table 2). Apart from reducing systemic immune resistance to infection postoperatively, ${ }^{11}$ gynecologic and genitourinary surgical procedures are known to cause postoperative bacteremia despite prophylactic antibiotics, increasing the risk of bone and joint infection. ${ }^{12,13}$ Furthermore, pregnancy and laparoscopic surgery are associated with an increase in intra-abdominal pressure (IAP). An increase in IAP can lead to increased pressure in the venous system of the spine via valveless intraabdominal lumbar veins and increased intrathoracic pressure, causing a decrease in venous drainage from the central nervous system via the jugular system. ${ }^{23}$ Hence, a combination of an altered immune response, postoperative bacteremia, and alteration in venous pressure gradient with the rise in IA,P which may promote the venous spread of 
infection, increases the risk of spinal infection in patients who have undergone nonspinal surgical procedures. $^{11-14,24}$

Our study has a few limitations. The treatment outcomes achieved in the current series at the end of 12 months need further validation in a larger study population. Furthermore, we did not aim to investigate the risk factors for developing PS following nonspinal surgery because of the small numbers in the current series. Most patients in the current series underwent surgeries with "clean" and "clean-contaminated" surgical wound categories (Table 2), received adequate perioperative antibiotic prophylaxis, and did not report surgical site infection in the postoperative period, and most of the patients had no comorbidities. This indicates that the general health and nutrition of the patient may have played an important role in increasing their risk for developing PS after their primary nonspinal surgery. A study by Lalueza et $\mathrm{al}^{25}$ investigated risk factors for metastatic osteoarticular infections after $S$ aureus bacteremia and reported that patients with osteoporosis and preexisting osteoarticular disease were at high risk for developing a metastatic osteoarticular infection. Hence, risk factors that may put patients who are undergoing nonspinal surgery at risk for PS need further investigation with a larger multicenter study. To the best of our knowledge, the current study is the largest series of patients with PS developed following nonspinal surgery with treatment outcomes reported for 12 months. This series highlights an important "at-risk" patient population who can develop PS.

\section{CONCLUSION}

With most patients with PS following nonspinal surgeries treated with conservative management, excellent clinical outcomes were achieved in all patients at 12 months after treatment. Pyogenic spondylodiscitis following nonspinal surgery requires a high degree of suspicion for early diagnosis and treatment. The diagnosis of PS should be considered in patients presenting with LBP with a recent history of undergoing a nonspinal surgical procedure. Early diagnosis and treatment can help achieve excellent clinical outcomes in these patients. Further studies are required to determine risk factors and possible perioperative precautions that can be taken to prevent PS in patients who undergo nonspinal surgeries.

\section{REFERENCES}

1. Kehrer M, Pedersen C, Jensen TG, Lassen AT. Increasing incidence of pyogenic spondylodiscitis: a 14-year populationbased study. J Infect. 2014;68(4):313-320. doi:10.1016/j.jinf. 2013.11.011

2. Jensen AG, Espersen F, Skinhøj P, Rosdahl VT, FrimodtMøller N. Increasing frequency of vertebral osteomyelitis following Staphylococcus aureus bacteraemia in Denmark 1980-1990. J Infect. 1997;34(2):113-118. doi:10.1016/s01634453(97)92395-1

3. Madhavan K, Chieng LO, Armstrong VL, Wang MY. Spondylodiscitis in end-stage renal disease: a systematic review. $J$ Neurosurg Spine. 2019;15:1-9. doi:10.3171/2018.9. SPINE18824

4. Tschugg A, Lener S, Hartmann S, Rietzler A, Neururer S, Thomé C. Primary acquired spondylodiscitis shows a more severe course than spondylodiscitis following spine surgery: a single-center retrospective study of 159 cases. Neurosurg Rev. 2018;41(1):141-147. doi:10.1007/s10143-017-0829-9

5. Pandita N, Paul S, Yadav G, Kalia RB, Kandwal P. Evaluation of challenges in diagnosis of spontaneous subacute pyogenic spondylodiscitis in immunocompetent patients: experiences from a tertiary care center. Asian Spine $J$. 2019;13(4):621-629. doi:10.31616/asj.2018.0220

6. Qu DC, Chen HB, Yang MM, Zhou HG. Management of lumbar spondylodiscitis developing after laparoscopic sacrohysteropexy with a mesh: a case report and review of the literature. Medicine (Baltimore). 2019;98(49):e18252. doi:10. 1097/MD.0000000000018252

7. Pasquer A, Djeudji F, Pechoux A, Barth X. Spondylodiscitis after rectopexy: diagnostic of a rare complication. Tech Coloproctol. 2017;21(1):63-64. doi:10.1007/s10151-016-1566-8

8. Li CC, Li CZ, Wu ST, Cha TL, Tang SH. Spondylodiscitis with epidural and psoas muscle abscesses as complications after transrectal ultrasound-guided prostate biopsy: report of a rare case. Eur J Case Rep Intern Med. 2017;4(8):000694. doi:10.12890/2017_000694

9. Mandegaran R, Tang CSW, Pereira EAC, Zavareh A. Spondylodiscitis following endovascular abdominal aortic aneurysm repair: imaging perspectives from a single centre's experience. Skeletal Radiol. 2018;47(10):1357-1369. doi: 10. 1007/s00256-018-2939-z

10. Thomson S, Alibhai K, Winkelaar G, et al. Case report of vertebral osteomyelitis and mycotic abdominal aortic aneurysm caused by Scedosporium apiospermum in a lung transplant patient with cystic fibrosis. Transplant Proc. 2015;47(1):204-209. doi:10.1016/j.transproceed.2014.07.072

11. Cui P, Fang X. Pathogenesis of infection in surgical patients. Curr Opin Crit Care. 2015;21(4):343-350. doi:10.1097/ MCC.0000000000000227

12. Woodd SL, Montoya A, Barreix M, et al. Incidence of maternal peripartum infection: a systematic review and metaanalysis. PLoS Med. 2019;16(12):e1002984. doi:10.1371/ journal.pmed.1002984

13. Mohee AR, Gascoyne-Binzi D, West R, Bhattarai S, Eardley I, Sandoe JA. Bacteraemia during transurethral resection of the prostate: what are the risk factors and is it more common than we think? PLoS One. 2016;11(7):e0157864. doi:10.1371/journal.pone.0157864

14. Duarte RM, Vaccaro AR. Spinal infection: state of the 
art and management algorithm. Eur Spine J. 2013;22(12):27872799. doi:10.1007/s00586-013-2850-1

15. Lener S, Hartmann S, Barbagallo GMV, Certo F, Thomé C, Tschugg A. Management of spinal infection: a review of the literature. Acta Neurochir (Wien). 2018;160(3):487-496. doi:10.1007/s00701-018-3467-2

16. Yeom JA, Lee IS, Suh HB, Song YS, Song JW. Magnetic resonance imaging findings of early spondylodiscitis: interpretive challenges and atypical findings. Korean $J$ Radiol. 2016;17(5):565-580. doi:10.3348/kjr.2016.17.5.565

17. Kumar Y, Gupta N, Chhabra A, Fukuda T, Soni N, Hayashi D. Magnetic resonance imaging of bacterial and tuberculous spondylodiscitis with associated complications and non-infectious spinal pathology mimicking infections: a pictorial review. BMC Musculoskelet Disord. 2017;18(1):244. doi:10. 1186/s12891-017-1608-z

18. Homagk L, Homagk N, Klauss JR, Roehl K, Hofmann GO, Marmelstein D. Spondylodiscitis severity code: scoring system for the classification and treatment of non-specific spondylodiscitis. Eur Spine J. 2016;25(4):1012-1020. doi:10. 1007/s00586-015-3936-8

19. Mannion AF, Vila-Casademunt A, Domingo-Sàbat M, et al. The Core Outcome Measures Index (COMI) is a responsive instrument for assessing the outcome of treatment for adult spinal deformity. Eur Spine J. 2016;25(8):2638-2648. doi:10.1007/s00586-015-4292-4

20. Mangram AJ, Horan TC, Pearson ML, Silver LC, Jarvis WR. Guideline for Prevention of Surgical Site Infection, 1999. Centers for Disease Control and Prevention (CDC) Hospital Infection Control Practices Advisory Committee. Am J Infect Control. 1999;27(2):97-132. doi:10.1016/S0196-6553(99)70088$\mathrm{X}$

21. Pola E, Autore G, Formica VM, et al. New classification for the treatment of pyogenic spondylodiscitis: validation study on a population of 250 patients with a follow-up of 2 years. Eur Spine J. 2017;26(suppl 4):479-488. doi:10.1007/s00586-0175043-5

22. Sur A, Tsang K, Brown M, Tzerakis N. Management of adult spontaneous spondylodiscitis and its rising incidence. Ann $R$ Coll Surg Engl. 2015;97(6):451-455. doi:10.1308/rcsann.2015. 0009
23. Giordan E, Marton E, Scotton G, Canova G. Outcomes and risk factors for spontaneous spondylodiscitis: case series and meta-analysis of the literature. J Clin Neurosci. 2019;68(10):179-187. doi: 10.1016/j.jocn.2019.06.040

24. Depauw PRAM, Groen RJM, Van Loon J, Peul WC, Malbrain MLNG, De Waele JJ. The significance of intraabdominal pressure in neurosurgery and neurological diseases: a narrative review and a conceptual proposal. Acta Neurochir (Wien). 2019;161(5):855-864. doi:10.1007/s00701-019-03868-7

25. Lalueza A, Morales-Cartagena A, Chaves F, et al. Risk factors for metastatic osteoarticular infections after a long follow-up of patients with Staphylococcus aureus bacteraemia. Clin Microbiol Infect. 2015;21(11):1010.e1-5. doi:10.1016/j.cmi. 2015.06.025

Disclosures and COI: No benefits or funds were received in support of this study by any of the authors. This study has been approved by an Institutional Review Board and ethics committee and has been performed in accordance with the ethical standards as laid down in the 1964 Declaration of Helsinki and its later amendments or comparable ethical standards.

Corresponding Author: Ghazwan A. Hasan, Department of Orthopaedic Surgery, Al-Kindi Teaching Hospital, Al-Qasim ibn Al-Hasan Expressway, Baghdad, Iraq. Phone: (964) 7700447666; Email: dr.bayaty@gmail.com.

Published 1 June 2021

This manuscript is generously published free of charge by ISASS, the International Society for the Advancement of Spine Surgery. Copyright (C) 2021 ISASS. To see more or order reprints or permissions, see http://ijssurgery.com. 